\title{
Parton Energy Loss in Generalized High Twist Approach
}

\author{
Yuan-Yuan Zhang* \\ Central China Normal University \\ E-mail: yuanyuanzhang@mails.ccnu.edu.cn \\ Guang-You Qin \\ Central China Normal University \\ E-mail: guangyou.qinemail.ccnu.edu.cn
}

\section{Xin-Nian Wang}

Lawrence Berkeley National Laboratory, Central China Normal University

E-mail: xnwang@lbl.gov

\begin{abstract}
The High Twist approach has been used to calculate parton energy loss and the medium modified fragmentation function due to multiple parton scattering. We revisit this problem for the deeply inelastic scattering (DIS) process with a Generalized High Twist approach. In this approach, the differential radiated gluon number distribution can be expressed in terms of the unintegrated gluon distribution function, or the transverse momentum dependent jet transport coefficient. The radiated gluon spectrum can be calculated without soft gluon and static medium approximation. In these limits, one can recover the GLV result in first order opacity approximation.
\end{abstract}

International Conference on Hard and Electromagnetic Probes of High-Energy Nuclear Collisions 30 September - 5 October 2018

Aix-Les-Bains, Savoie, France

${ }^{*}$ Speaker. 


\section{Introduction}

In high energy collisions, the energetic parton, the hard probe, travels through the medium. The medium can be hot and dense Quark Gluon Plasma (QGP) which is created in ultra-relativistic heavy-ion collisions. The medium can also be cold nuclei, like in the electron nucleus deeply inelastic scattering (DIS) process. The parton loses energy along its way when it travels through the medium. The energy loss mechanism (or jet quenching) is sensitive to medium properties. One usually categorizes the parton energy loss as two kinds, collisional energy loss and radiative energy loss. In our study, we focus on the parton radiative energy loss in the electron nucleus DIS process.

There are already several approaches to deal with radiative energy loss, like BDMPS-Z, GLV, AMY, SCET and High Twist (see Ref. [1] for a review of these approaches). There are several assumptions in these approaches. The first, as used by the original BDMPS-Z and GLV approaches, is that scattering centers are static, i.e. there is no energy transfer in the parton-medium scattering. While for dynamic scattering centers, there is both momentum and energy transfer during scattering. The second assumption is about the radiated gluon which can be soft or hard. The third assumption is about the relative magnitude of transverse momentum transfer $k_{\perp}$ during scattering and radiated gluon transverse momentum $l_{\perp}$. The High Twist approach assumes $l_{\perp} \gg k_{\perp}$. There are already some efforts $[2,3,4]$ to extend the assumptions made by different approaches to more realistic ones. The motivation of our study is to calculate the radiative energy loss assuming dynamic scattering centers beyond soft radiated gluon approximation and allowing $l_{\perp} \sim k_{\perp}$.

This short manuscript is organized as follows. The introduction on the semi-inclusive DIS process used in High Twist approach is given in section 2. Section 3 contains the details of our Generalized High Twist approaches, including the discussion on the unintegrated gluon distribution function and on the jet transport parameter. The approximations one can make for our approach are also discussed here. The summary is given in section 4 .

\section{High Twist Approach}

The process for radiative energy loss calculation in the High Twist approach is the semiinclusive DIS process. The lepton scatters with the nuclei, and one identifies the scattered lepton and one final hadron. The cross section for this process can be written as the product of the leptonic tensor $L_{\mu \nu}$ and the hadronic tensor $W^{\mu \nu}$. The hadronic tensor is of our interest. For the leading order hadronic tensor, one can use the important factorization theorem to separate the nonperturbative part (parton distribution function $f_{q}^{A}(x)$, fragmentation function $D_{q \rightarrow h}\left(z_{h}\right)$ ) from the perturbative part (hard scattering $H_{(0)}^{\mu v}(x)$ ).

$$
\frac{d W_{S(0)}^{\mu v}}{d z_{h}}=\int d x f_{q}^{A}(x) H_{(0)}^{\mu v}(x) D_{q \rightarrow h}\left(z_{h}\right) .
$$

For the final state parton which undergoes the medium induced radiation, the problem we focus on is the factorization of medium induced radiation for the higher twist hadronic tensor. The High Twist approach [5] performs the collinear factorization. After obtaining the arranged expression 
for the hadronic tensor

$$
\begin{aligned}
\frac{d W_{D(1) q}^{\mu v}}{d z_{h}}= & \int_{z_{h}}^{1} \frac{d z}{z} D_{q \rightarrow h}\left(z_{h} / z\right) \int \frac{d y^{-}}{2 \pi} d y_{1}^{-} d y_{2}^{-} \frac{d^{2} y_{1 \perp}}{(2 \pi)^{2}} d^{2} y_{2 \perp} d^{2} k_{\perp} e^{-i \vec{k}_{\perp} \cdot\left(\vec{y}_{1 \perp}-\vec{y}_{2 \perp}\right)} \\
& \frac{1}{2}\left\langle A\left|\bar{\psi}_{q}(0) \gamma^{+} A^{+}\left(y_{2}^{-}, \vec{y}_{2 \perp}\right) A^{+}\left(y_{1}^{-}, \vec{y}_{1 \perp}\right) \psi_{q}\left(y^{-}\right)\right| A\right\rangle H_{D}^{\mu v}\left(k_{\perp}, y^{-}, y_{1}^{-}, y_{2}^{-}, p, q, z\right),
\end{aligned}
$$

one takes the approximation that $l_{\perp} \gg k_{\perp}$, and performs a collinear expansion of the hard part. It is the Taylor expansion of the hard part in terms of $k_{\perp}$ :

$$
H_{D}^{\mu v}\left(k_{\perp}, y^{-}, y_{1}^{-}, y_{2}^{-}, p, q, z\right)=H_{D}^{\mu v}\left(k_{\perp}=0\right)+\left.\frac{\partial H_{D}^{\mu v}}{\partial k_{\perp}^{\alpha}}\right|_{k_{\perp}=0} k_{\perp}^{\alpha}+\left.\frac{1}{2} \frac{\partial^{2} H_{D}^{\mu v}}{\partial k_{\perp}^{\alpha} \partial k_{\perp}^{\beta}}\right|_{k_{\perp}=0} k_{\perp}^{\alpha} k_{\perp}^{\beta}+\cdots
$$

The first term contributes to the gauge link of the initial quark distribution function. The $k_{\perp}^{\alpha}$ term contributes zero for unpolarized beams, so the main contribution to the hadronic tensor comes from the quadratic term in $k_{\perp}$.

\section{Generalized High Twist Approach}

The Generalized High Twist approach relaxes the approximation of $l_{\perp} \gg k_{\perp}$, allowing $l_{\perp} \sim k_{\perp}$. Without collinear expansion, we factorize the quark distribution and the gluon distribution function directly. The hadronic tensor can be written as follows,

$$
\begin{aligned}
\frac{d W_{D(1) q}^{\mu v}}{d z_{h}} \sim & \frac{\alpha_{s}}{2 \pi} C_{F} \frac{2 \pi \alpha_{s}}{N_{c}} \frac{1+z^{2}}{1-z} H_{(0)}^{\mu v}\left(x_{B}\right) \otimes \rho\left(y_{1}^{-}, \vec{y}_{1 \perp}\right) \otimes \frac{D_{q \rightarrow h}\left(z_{h} / z\right)}{z} \otimes f_{q}^{A}\left(x_{B}\right) \\
& \otimes \frac{\pi}{\left[\vec{l}_{\perp}-(1-z) \vec{k}_{\perp}\right]^{2}} \frac{\phi\left(x_{L}+x_{D}, \vec{k}_{\perp}\right)}{k_{\perp}^{2}}
\end{aligned}
$$

the convolution of splitting function, hard part, nucleon density at $\left(y_{1}^{-}, y_{1 \perp}\right)$, fragmentation function $D_{q \rightarrow h}\left(z_{h} / z\right)$, quark distribution function $f_{q}^{A}\left(x_{B}\right)$ and transverse momentum dependent (TMD) gluon distribution function $\phi\left(x_{L}+x_{D}, \vec{k}_{\perp}\right) .\left(x_{B}=\frac{Q^{2}}{2 p^{+} q^{-}}, x_{L}=\frac{l_{\perp}^{2}}{2 p^{+} q^{-} z(1-z)}, x_{D}=\frac{k_{\perp}^{2}-2 \vec{k}_{\perp} \cdot l_{\perp}}{2 p^{+} q^{-} z}\right)$. This is the hadronic tensor for one central cut Feynman diagram. The TMD gluon distribution function $\phi\left(x, \vec{k}_{\perp}\right)$, which appears in the factorized hadronic tensor above, emerges from the TMD transport parameter $\hat{q}\left(k_{\perp}\right)$. The definition of transport parameter is the product of the color source density and the average $k_{\perp}$ broadening per scattering. Considering the scattering of a parton (momentum $l$ ) with a nucleon (momentum $p$ ), we calculate the averaged cross section $\langle d \sigma\rangle$ and obtain $\hat{q}$ :

$$
\hat{q}=\rho \int d k_{\perp}^{2} \frac{\langle d \sigma\rangle}{d k_{\perp}^{2}} k_{\perp}^{2} \equiv \int \frac{d k_{\perp}^{2}}{(2 \pi)^{2}} \hat{q}\left(\vec{k}_{\perp}\right)=\int \frac{d k_{\perp}^{2}}{(2 \pi)^{2}} \int d x \delta\left(x-\frac{k_{\perp}^{2}}{2 p^{+} l^{-}}\right) \frac{4 \pi \alpha_{s} C_{2}(R)}{N_{c}^{2}-1} \rho(y) \phi\left(x, \vec{k}_{\perp}\right) .
$$

One can find the definition of the TMD transport parameter $\hat{q}\left(\vec{k}_{\perp}\right)$, and the TMD gluon distribution also emerges naturally here

$$
\phi\left(x, \vec{k}_{\perp}\right)=\int \frac{d y^{-}}{2 \pi p^{+}} \int d^{2} y_{\perp} e^{-i x p^{+} y^{-}+\vec{k}_{\perp} \cdot \vec{y}_{\perp}}\left\langle p\left|F_{\alpha}{ }^{+}\left(y^{-}, \vec{y}_{\perp}\right) F^{+\alpha}\left(0, \overrightarrow{0}_{\perp}\right)\right| p\right\rangle .
$$


Both the TMD transport parameter $\hat{q}\left(\vec{k}_{\perp}\right)$ and the TMD gluon distribution $\phi\left(x, \vec{k}_{\perp}\right)$ depend on the parton energy $l^{-}$and the medium color source (here the nucleon) energy $p^{+}$through the parameter $x$. Also the energy transfer during scattering is represented by $x$.

Summing up the central cut diagrams ( 9 diagrams), left cut diagrams (7 diagrams) and right cut diagrams ( 7 diagrams), we can get the hadronic tensor for all the medium induced radiation scenarios of $\alpha_{s}^{2}$ order. The central cut diagrams have the final state cutting line in between of the two gluon lines. The right/left cut diagrams have the final state cutting line on the right/left of two gluon lines. When collecting terms, there are some negligible terms, called contact terms. The integration region of contact terms is constrained in $0<y_{2}^{-}<y_{1}^{-}<y^{-}$. This means the quark distribution function and TMD gluon distribution function originate from the same gluon. While for other terms, the quark and gluon originate from different nucleons, this gives the nuclear enhancement factor. In this case, these contact terms are negligible compared to other terms.

After setting aside contact terms, we get the radiated gluon spectrum from the hadronic tensor

$$
\frac{d W_{D(1) g}^{\mu v}}{d z_{h}}=\int d x f_{q}^{A}\left(x_{B}\right) H_{(0)}^{\mu v} \int \frac{d z}{z} D_{g \rightarrow h}\left(z_{h} / z\right) \int d l_{\perp}^{2} \frac{d N}{d l_{\perp}^{2} d z} .
$$

The gluon spectrum can be expressed in terms of the TMD gluon distribution

$$
\frac{d N}{d l_{\perp}^{2} d z}=\frac{\pi}{f_{q}^{A}\left(x_{B}\right)} \frac{\alpha_{s}}{2 \pi} \frac{1+(1-z)^{2}}{z} \frac{2 \pi \alpha_{s}}{N_{c}} \int \frac{d^{2} k_{\perp}}{(2 \pi)^{2}} \int_{y^{-}}^{\infty} d y_{1}^{-} \int d^{2} \vec{y}_{1 \perp} \rho\left(y_{1}^{-}, \vec{y}_{1 \perp}\right)\left[\tilde{H}_{C}^{D}+\frac{1}{2} \tilde{H}_{L}^{D}+\frac{1}{2} \tilde{H}_{R}^{D}\right]
$$

Here $\mathrm{z}$ is the radiated gluon momentum fraction from the parent parton, and $x_{T}=x_{D}(z \rightarrow 1-z)$. After we have obtained the general result for the gluon spectrum, there are several approximations one can take.

1.) Static scattering center approximation, which means no energy transfer for parton medium scattering. The TMD gluon distributions do not have $x$ dependence, and also the quark distributions all reduce to $f_{q}^{A}\left(x_{B}\right)$. This indicates $x_{B} \gg x_{L}, \frac{x_{T}}{z}$. One can see the expression has been simplified.

$$
\begin{aligned}
\frac{d N_{\text {static }}}{d l_{\perp}^{2} d z}= & \pi \frac{\alpha_{s}}{2 \pi} \frac{1+(1-z)^{2}}{z} \frac{2 \pi \alpha_{s}}{N_{c}} \int \frac{d^{2} k_{\perp}}{(2 \pi)^{2}} \int d y_{1}^{-} \int d^{2} \vec{y}_{1 \perp} \rho\left(y_{1}^{-}, \vec{y}_{1 \perp}\right)\left[\frac{C_{A}}{\left(l_{\perp}-k_{\perp}\right)^{2}}-\frac{C_{A}}{l_{\perp}^{2}}+\frac{C_{F}}{l_{\perp}^{2}}\right. \\
& +C_{A} \frac{\vec{k}_{\perp} \cdot \vec{l}_{\perp}}{l_{\perp}^{2}\left(\vec{l}_{\perp}-\vec{k}_{\perp}\right)^{2}}+\frac{C_{F}}{\left[\vec{l}_{\perp}-z \vec{k}_{\perp}\right]^{2}}+\frac{1}{N} \frac{\vec{l}_{\perp} \cdot\left[\vec{l}_{\perp}-z \vec{k}_{\perp}\right]}{l_{\perp}^{2}\left[\vec{l}_{\perp}-z \vec{k}_{\perp}\right]^{2}}-C_{A} \frac{\left(\vec{l}_{\perp}-\vec{k}_{\perp}\right) \cdot\left[\vec{l}_{\perp}-z \vec{k}_{\perp}\right]}{\left(l_{\perp}-k_{\perp}\right)^{2}\left[\vec{l}_{\perp}-z \vec{k}_{\perp}\right]^{2}} \\
& +\left(-\frac{2 C_{A}}{\left(\vec{l}_{\perp}-\vec{k}_{\perp}\right)^{2}}+C_{A} \frac{\vec{l}_{\perp} \cdot\left(\vec{l}_{\perp}-\vec{k}_{\perp}\right)}{l_{\perp}^{2}\left(\vec{l}_{\perp}-\vec{k}_{\perp}\right)^{2}}+C_{A} \frac{\left(\vec{l}_{\perp}-\vec{k}_{\perp}\right) \cdot\left[\vec{l}_{\perp}-z \vec{k}_{\perp}\right]}{\left(l_{\perp}-k_{\perp}\right)^{2}\left[\vec{l}_{\perp}-z \vec{k}_{\perp}\right]^{2}}\right) \cos \left[\left(x_{L}+\frac{x_{T}}{z}\right) p^{+} y_{1}^{-}\right] \\
& +\left(-2 C_{F} \frac{1}{l_{\perp}^{2}}-\frac{1}{N} \frac{\vec{l}_{\perp} \cdot\left[\vec{l}_{\perp}-z \vec{k}_{\perp}\right]}{l_{\perp}^{2}\left[\vec{l}_{\perp}-z \vec{k}_{\perp}\right]^{2}}+\frac{C_{A}}{l_{\perp}^{2}}\right) \cos \left[x_{L} p^{+} y_{1}^{-}\right] \frac{\phi\left(0, \vec{k}_{\perp}\right)}{k_{\perp}^{2}}
\end{aligned}
$$

2.) Soft radiated gluon approximation, this means the radiated gluon momentum fraction $z \rightarrow 0$. Under this approximation, the gluon spectrum does not have $\mathrm{z}$ dependence, except in quark, gluon distribution function and phase factor.

3.) Static scattering center + Soft radiated gluon approximation. One should notice $x_{B} \gg x_{L}, \frac{x_{T}}{z}$ 
should also be valid when $z \rightarrow 0$. The gluon spectrum simplifies to

$$
\begin{aligned}
\frac{d N_{\text {static }+ \text { soft }}}{d l_{\perp}^{2} d z}= & \pi \frac{\alpha_{s}}{2 \pi} \frac{1+(1-z)^{2}}{z} \frac{2 \pi \alpha_{s}}{N_{c}} \int \frac{d^{2} k_{\perp}}{(2 \pi)^{2}} \int d y_{1}^{-} \int d^{2} \vec{y}_{1 \perp} \rho\left(y_{1}^{-}, \vec{y}_{1 \perp}\right) \\
& C_{A} \frac{2 \vec{k}_{\perp} \cdot \vec{l}_{\perp}}{l_{\perp}^{2}\left(\vec{l}_{\perp}-\vec{k}_{\perp}\right)^{2}}\left(1-\cos \left[\left(x_{L}+\frac{x_{T}}{z}\right) p^{+} y_{1}^{-}\right]\right) \frac{\phi\left(0, \vec{k}_{\perp}\right)}{k_{\perp}^{2}}
\end{aligned}
$$

Inside the gluon spectrum, there is one unknown quantity, the TMD gluon distribution function $\phi\left(0, \vec{k}_{\perp}\right)$. The $\phi\left(x, \vec{k}_{\perp}\right)$ is a non-perturbative quantity, one can calculate it using many different methods, such as Hard Thermal Loop theory. In this special case, when $x=0$, we can use the static potential model to calculate $\phi\left(0, \vec{k}_{\perp}\right)$. As discussed earlier, the TMD gluon distribution function emerges in the transport parameter $\hat{q}$. In the static potential model, one calculates the cross section of elastic scattering between partons, and obtains $\hat{q}$. Comparing with the Eq. 3.2, one can get the expression of $\phi\left(0, \vec{k}_{\perp}\right)$.

$$
\phi\left(0, \vec{k}_{\perp}\right)=C_{2}(T) \frac{4 \alpha_{s}}{k_{\perp}^{2}+\mu_{0}^{2}} .
$$

Substitute it into the gluon spectrum, one can find this gluon spectrum agrees with the GLV approach result at first order in opacity[6]. Only the arguments in the cosine function are expressed in light cone coordinates for our result, while in Cartesian coordinates for GLV result, but they are the same.

\section{Summary}

In our study, we investigate the radiative energy loss in the semi-inclusive DIS process. The approach we used is the Generalized High Twist approach. Besides the dynamic scattering center, and beyond soft radiated gluon approximation, we further relax the $l_{\perp} \gg k_{\perp}$ approximation. The TMD gluon distribution function $\phi\left(x, \vec{k}_{\perp}\right)$ is introduced, and it relates to the TMD transport parameter $\hat{q}\left(\vec{k}_{\perp}\right)$. We also show, under some approximations, the Generalized High Twist approach result can be reduced to the GLV formalism result.

\section{References}

[1] Qin, Guang-You and Wang, Xin-Nian, Jet quenching in high-energy heavy-ion collisions, in Quark-Gluon Plasma 5, pp. 309-372. World Scientific, 2016.

[2] Djordjevic, Magdalena and Heinz, Ulrich, Radiative Energy Loss in a Finite Dynamical QCD Medium, Physical Review Letters 101 (2008) .

[3] Blagojevic, Bojana and Djordjevic, Magdalena and Djordjevic, Marko, Calculating hard probe radiative energy loss beyond soft-gluon approximation: how valid is the approximation?, arXiv preprint arXiv:1804.07593 (2018) .

[4] Ovanesyan, Grigory and Vitev, Ivan, An effective theory for jet propagation in dense QCD matter: jet broadening and medium-induced bremsstrahlung, Journal of High Energy Physics 2011 (2011) .

[5] Wang, Xin-Nian and Guo, Xiaofeng, Multiple parton scattering in nuclei: Parton energy loss, Nuclear Physics A 696 (2001) 788.

[6] Gyulassy, M. and Levai, P. and Vitev, I., Non-Abelian Energy Loss at Finite Opacity, Physical Review Letters 85 (2000) 5535. 